\title{
Fault Diagnosis of a Wind Farm using Interval Parity Equations ${ }^{1}$ J. Blesa $^{* * * *}$, P. Jiménez ${ }^{* * * *}$, D. Rotondo*, F. Nejjari*, V. Puig ${ }^{* * * *}$ \\ * Automatic Control Department (ESAII), Technical University of Catalonia (UPC) \\ Pau Gargallo 5, 08028 Barcelona, Spain. (e-mail: joaquim.blesa@upc.edu) \\ **Institut de Robòtica I Informàtica Industrial (CSIC-UPC) \\ Carrer Llorens i Artigues, 4-6, 08028 Barcelona, Spain
}

\begin{abstract}
In this paper, the problem of fault diagnosis of a wind farm is addressed using interval parity equations. Fault detection is based on the use of parity equations and unknown but bounded description of the noise and modeling errors. The fault detection test is based on checking the consistency between the measurements and the model by finding if the formers are inside the interval prediction bounds. The fault isolation algorithm is based on analyzing the observed fault signatures on-line, and matching them with the theoretical ones obtained using structural analysis. Finally, the proposed approach is tested using the wind farm benchmark proposed in the context of the wind farm FDI/FTC competition.
\end{abstract}

Keywords: Fault Diagnosis, Wind farm, Interval parity Equations

\section{INTRODUCTION}

Wind energy has gained a big interest these last years as one of the most promising and abundant sources for green and sustainable energy. However, wind farms are characterized by high manufacturing and maintenance costs that bring challenging reliability and lifetime issues. Introducing fault diagnosis and fault tolerant control (FTC) is considered a suitable way for improving their reliability and reducing their maintenance.

In recent years, the problem of fault diagnosis and FTC of wind turbines has become an important topic of research. In Odgaard et al. (2013), a benchmark model for fault diagnosis and FTC of wind turbines has been proposed and results obtained using several approaches are compared. This benchmark relies on a realistic three blade horizontal variable speed wind turbine with a full scale converter coupling and a rated power of $4.8 \mathrm{MW}$. In some of the the works summarised in Odgaard et al. (2013), the fault detection problem has been addressed using model-based approaches that are based on analytical redundancy and checking the consistency of the observed behavior with respect to the system one. This consistency checking is based on computing the difference, called residual, between the value predicted from the model and the real value measured by the sensors. In case a discrepancy is detected, a fault in the system is indicated. Otherwise, it is considered that the system is working properly.

In the case of LTI systems, model-based fault detection theory is well developed (Blanke et al. 2006; Isermann, 2005). However, since these models are based on LTI lumped parameter models, they are valid only around a given operating point. In order to use these simplified models in large operating conditions, the influence of the operating point in the parameters of the LTI model should be taken into account in some way. In this paper, the use of non-linear parameter varying (NLPV) models is proposed to consider the variation of the parameters with the operating point. This type of models adds a non-linear dynamic map to the classical LPV model, which takes into account the scheduling variables available for measurement, resulting in a hybrid linear/nonlinear model. In this way, the advantages of a parametrically varying structure and the generality of the NARMAX (nonlinear autoregressive exogenous moving average) class (Previdi and Lovera, 2004) are combined.

Fault detection methods based on mathematical models are always affected by modeling errors. These modeling errors introduce uncertainty in the model and interfere with the fault detection. A fault detection algorithm able to handle uncertainty is called robust, and its robustness represents the degree of sensitivity to faults compared to the degree of sensitivity to uncertainty (Chen and Patton, 1999). In this paper, the uncertainty will be located in the parameters and in the delay of the NLPV model, bounding their values by intervals. One of the FDI approaches, known as passive, which enhances the robustness of the fault detection system at the decision-making stage, is based on using an adaptive threshold (Puig et al. 2008; Fagasaran et al.2004; Sainz et al. 2002) generated by considering the set of model responses obtained by varying the uncertain parameters within their intervals.

The aim of this paper is to address the problem of fault diagnosis of a wind farm using interval parity equations (Patton and Chen, 1994). Fault detection is based on the use of parity equations and unknown but bounded description of the noise and modeling errors. The fault detection test is based on checking consistency between the measurements

\footnotetext{
1 This work has been funded by the Spanish MINECO through the project CYCYT SHERECS (ref. DPI2011-26243), by the European Commission through contract i-Sense (ref. FP7-ICT-2009-6-270428) and by AGAUR through the contract FI-DGR 2013 (ref. 2013FIB00218).
} 
and the model, finding out if the formers are inside the interval prediction bounds. The fault isolation algorithm is based on analyzing the observed fault signatures on-line, and matching them with the theoretical ones obtained using structural analysis. The proposed approach is tested using the wind farm benchmark proposed in the context of the wind farm FDI/FTC competition (Odgaard and Stoustrup, 2013).

The paper is organized as follows. In Section 2, a method using interval parity equations for fault detection is introduced. Section 3 presents a method for the calibration of interval models. In Section 4, the wind farm benchmark is presented and the fault diagnosis approach based on interval parity equations is applied. The results obtained applying the proposed method to the wind farm benchmark, are shown in Section 5. Finally, in Section 6, the main conclusions are presented.

\section{FAULT DETECTION USING INTERVAL PARITY EQUATIONS}

\subsection{Interval model}

Let us assume that the system can be expressed by means of the following NLPV model in regression form:

$$
y(k)=F\left(k, \boldsymbol{\theta}\left(\mathbf{p}_{k}\right)\right)+e(k)=\hat{y}(k)+e(k)
$$

where:

- $F\left(k, \theta\left(\mathbf{p}_{k}\right)\right)$ is the regressor function that computes the estimation $\hat{y}(k)$ which, in general, is assumed to be nonlinear in the parameters $\boldsymbol{\theta}\left(\mathbf{p}_{k}\right)$ and can contain any function of inputs $u(k)$ and outputs $y(k)$;

- $\mathbf{p}_{k} \triangleq \mathbf{p}(k)$ is a vector of measurable process variables that defines the system operating point;

- $\boldsymbol{\theta}\left(\mathbf{p}_{k}\right) \in \boldsymbol{\Theta}_{k}$ is the parameter vector of dimensions $n_{\theta} \times 1$ whose values can vary according to the system operating point;

- $\boldsymbol{\Theta}_{k}$ is the set that bounds the parameter values, and can vary according to the system operating point as well. In particular, in this paper, the set of uncertain parameters is bounded by an interval box in the nominal parameter values:

$$
\boldsymbol{\Theta}_{k} \triangleq\left[\underline{\theta}_{1}\left(\mathbf{p}_{k}\right), \bar{\theta}_{1}\left(\mathbf{p}_{k}\right)\right] \times \cdots \times\left[\underline{\theta}_{n_{\theta}}\left(\mathbf{p}_{k}\right), \bar{\theta}_{n_{\theta}}\left(\mathbf{p}_{k}\right)\right]
$$

where:

$$
\underline{\theta}_{i}\left(\mathbf{p}_{k}\right) \triangleq \theta_{i}^{0}\left(\mathbf{p}_{k}\right)-\lambda_{i}(k) ; \bar{\theta}_{i}\left(\mathbf{p}_{k}\right) \triangleq \theta_{i}^{0}\left(\mathbf{p}_{k}\right)+\lambda_{i}(k) i=1, \ldots, n_{\theta},
$$

being $\theta_{i}^{0}\left(\mathbf{p}_{k}\right)$ the nominal parameter values that follow some known function $f\left(\mathbf{p}_{k}\right)$, and $\lambda_{i}(k) \geq 0$ the parameter uncertainties;

- $e(k)$ is an additive error term, which is unknown but assumed to be bounded by a constant $|e(k)| \leq \sigma$.

\subsection{Interval fault detection using parity equations}

The principle of model-based fault detection using parity equations is to test whether the measured input and output of the system are consistent with the behavior described by a model of the faultless system or not. If an inconsistency is detected, a fault is indicated. The residual usually describes the consistency check between the real behavior, $y(k)$, and the predicted one , $\hat{y}(k)$, as follows:

$$
r(k)=y(k)-\hat{y}(k)
$$

where $\hat{y}(k)=F\left(k, \boldsymbol{\theta}\left(\mathbf{p}_{k}\right)\right)$, considering the system described by (1).

Ideally, residuals should only be affected by the faults. However, the presence of disturbances, noise and modeling errors causes residuals to become non-zero, interfering with the detection of faults. Therefore, the fault detection procedure must be robust against these undesired effects (Chen and Patton, 1999). In this work, only noise and modeling errors will be considered in the robust fault detection method, following the passive approach recalled in the introduction.

In the case of modeling a dynamical system using the interval NLPV model (1), the predicted output behavior can be bounded at any iteration by an interval $[\underline{\hat{y}}(k), \overline{\hat{y}}(k)]$ computed by solving the two following optimization problems:

$$
\begin{aligned}
& \overline{\hat{y}}(k)=\max \left(F\left(k, \boldsymbol{\theta}\left(\mathbf{p}_{k}\right)\right)\right) \text { s.t. } \boldsymbol{\theta}\left(\mathbf{p}_{k}\right) \in \boldsymbol{\Theta}_{k} \\
& \underline{\hat{y}}(k)=\min \left(F\left(k, \boldsymbol{\theta}\left(\mathbf{p}_{k}\right)\right)\right) \text { s.t. } \boldsymbol{\theta}\left(\mathbf{p}_{k}\right) \in \boldsymbol{\Theta}_{k}
\end{aligned}
$$

Then, the fault detection test is based on propagating the parameter uncertainty and the error bounds to the residual (2) and checking if:

$$
0 \in[\underline{r}(k), \bar{r}(k)]=[y(k)-\overline{\hat{y}}(k)-\sigma, y(k)-\underline{\hat{y}}(k)+\sigma]
$$

or, alternatively:

$$
y(k) \in[\underline{\hat{y}}(k)-\sigma, \overline{\hat{y}}(k)+\sigma]
$$

holds or not. In case it does not hold, a fault can be indicated.

\subsection{Fault detection for uncertain varying time delay systems}

In case that (1) is used to represent a system with transport delay between input and output, this delay can also vary with the operating point and can be characterized by some measured variables. Considering such varying transport delay $\tau\left(\mathbf{p}_{k}\right)$ in the model (1), the output prediction can be expressed as:

$$
\hat{y}(k)=F\left(k, \boldsymbol{\theta}\left(\mathbf{p}_{k}\right), d\left(\mathbf{p}_{k}\right)\right)
$$

with $d\left(\mathbf{p}_{k}\right)=\left[\frac{\tau\left(\mathbf{p}_{k}\right)}{T_{s}}\right]$, where [ ] denotes the nearest integer and $T_{s}$ is the sampling time. Some uncertainty $\lambda_{\tau}$ in the delay is also considered such that:

$$
\tau\left(\mathbf{p}_{k}\right) \in\left[\tau_{0}\left(\mathbf{p}_{k}\right)-\lambda_{\tau}, \tau_{0}\left(\mathbf{p}_{k}\right)+\lambda_{\tau}\right]
$$


where the nominal delay satisfies: $\tau_{0}\left(\mathbf{p}_{k}\right)>\lambda_{\tau}$ and $\tau_{0}\left(\mathbf{p}_{k}\right), \lambda_{\tau} \in \mathbb{R}^{+}$.

Then, as was suggested in Blesa et al. (2010), the interval for the predicted output given by (6) can be expressed as follows:

$$
\begin{aligned}
& \overline{\hat{y}}(k)=\max _{\boldsymbol{\theta}\left(\mathbf{p}_{k}\right) \in \boldsymbol{\Theta}_{k}, d\left(\mathbf{p}_{k}\right) \in\left\{\underline{d\left(\mathbf{p}_{k}\right)}, \ldots, \overline{d\left(\mathbf{p}_{k}\right)}\right\}} F\left(k, \boldsymbol{\theta}\left(\mathbf{p}_{k}\right), d\left(\mathbf{p}_{k}\right)\right) \\
& \hat{y}(k)=\min _{\boldsymbol{\theta}\left(\mathbf{p}_{k}\right) \in \boldsymbol{\Theta}_{k}, d\left(\mathbf{p}_{k}\right) \in\left\{\underline{\left.d\left(\mathbf{p}_{k}\right), \ldots, \overline{d\left(\mathbf{p}_{k}\right)}\right\}} F\left(k, \boldsymbol{\theta}\left(\mathbf{p}_{k}\right), d\left(\mathbf{p}_{k}\right)\right)\right.}
\end{aligned}
$$

with:

$$
\underline{d\left(\mathbf{p}_{k}\right)}=\left[\frac{\tau_{0}\left(\mathbf{p}_{k}\right)-\lambda_{\tau}}{T_{s}}\right] \text { and } \overline{d\left(\mathbf{p}_{k}\right)}=\left[\frac{\tau_{0}\left(\mathbf{p}_{k}\right)+\lambda_{\tau}}{T_{s}}\right]
$$

\section{WIND FARM BENCHMARK}

\subsection{Wind farm model}

The wind farm benchmark is made up by 9 wind turbines in a square grid layout (Fig. 1). The distance between the wind turbines in both directions is $7 L$, where $L$ is the rotor diameter. Two measuring masts are located in front of the wind turbine, one for each possible wind direction considered in the benchmark. The distance between the measuring mast and the wind farm is $10 \mathrm{~L}$. Each turbine is a generic $4.8 \mathrm{MW}$ wind turbine, as the one described in Odgaard et al. (2013), and is numbered using the corresponding row and column in the wind farm. Each wind turbine is driven by the wind farm controller that provides a power reference.

The model consists of three parts: the wind \& wake model, wind turbine model and the wind farm controller. The details can be found in Odgaard et al. (2010) and Odgaard and Stoustrup (2013).

\subsection{Fault scenarios}

Three different faults are considered in the benchmark, affecting the three measured variables $P$ (power) , $\beta$ (pitch angle) and $\omega_{g}$ (angular velocity). The faults are hard to detect at a wind turbine level, but can be detected at the wind farm level comparing the performances of the other wind turbines.

Fault 1: debris build-up on the wind turbine blades, changing the aerodynamics of the wind turbine, lowering the maximum obtained power.

Fault 2: misalignment of one or more blades originated at the time of installation of the wind turbine.

Fault 3: change in the drive train damping due to wear and tear.

\subsection{Wind estimation}

The wind sequence considered in each wind turbine is estimated by considering the distance between the measuring mast and the wind turbine itself. The delay in the wind sequence is the distance divided by the mean wind velocity while the wake is modeled by a wind deficit between the wind turbines by a factor of 0.9 .

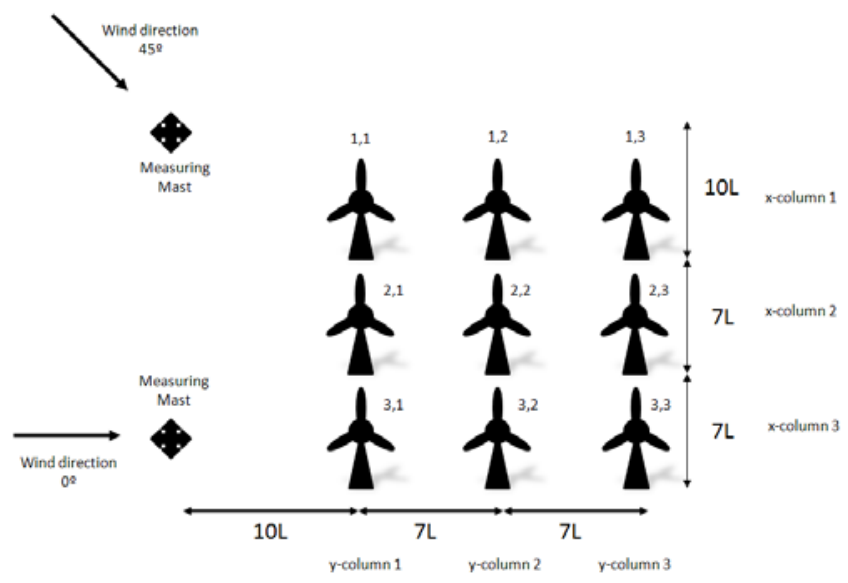

Fig. 1. Illustration of the layout of the example wind farm.

\section{FAULT DIAGNOSIS APPROACH}

\subsection{Residual generation}

The models considered for FDI in the wind farm take into account the temporal and spatial redundancy existing in the wind farm.

The temporal redundancy in each wind turbine can be taken into account by considering the estimated wind that each wind turbine receives and the estimated power, pitch angle and angular velocity, as follows:

$$
\begin{gathered}
\dot{P}_{i, j}(t)=f_{P_{i, j}}\left(P_{i, j}(t), v_{w_{i, j}}(t), P_{r_{i, j}}(t)\right) \\
\dot{\beta}_{i, j}(t)=f_{\beta_{i, j}}\left(\beta_{i, j}(t), v_{w_{i, j}}(t), P_{r_{i, j}}(t)\right) \\
\dot{\omega}_{g i, j}(t)=f_{\omega_{g i, j}}\left(\omega_{g i, j}(t), v_{w_{i, j}}(t), P_{r_{i, j}}(t)\right)
\end{gathered}
$$

where $f_{P_{i, j}}, f_{\beta_{i, j}}$ and $f_{\omega_{g i, j}}$ are non-linear functions, $P_{r_{i, j}}(t)$ is the control signal provided to the turbine $i, j$ by the wind farm controller and $v_{w_{i, j}}(t)$ is the wind speed at the turbine $i, j$, that can be estimated from the wind speed measured in a wind mast $v_{w}(t)$ with:

$$
v_{w_{i, j}}(t)=f_{v_{w, j}}\left(v_{w}\left(t-\tau_{i, j}(t)\right)\right)+e_{w_{i, j}}(t)=\hat{v}_{w_{i, j}}(t)+e_{w_{i, j}}(t)
$$

where $e_{w_{i, j}}(t)$ is the wind estimation error in turbine $i, j$ considered unknown but bounded $\left|e_{w_{i, j}}(t)\right| \leq \sigma_{w_{i, j}}$ and $\tau_{i, j}$ is the wind transport delay from the wind mast to the turbine $i, j$, that can be calculated as:

$$
\tau_{i, j}(t)=f\left(D_{i, j}, v_{w}^{0}(t)\right)+e_{\tau_{i, j}}(t), \quad\left|e_{\tau_{i, j}}(t)\right| \leq \lambda_{\tau_{i, j}}
$$

where $D_{i, j}$ is the distance from the wind mast to the turbine $i, j, v_{w}^{0}(t)$ is the mean wind speed at the wind mast point (see Fig. 1) obtained by filtering the wind measurement $v_{w}(t)$, and the bounded additive error $e_{\tau_{i, j}}(t)$ is the error in the delay estimation. In the wind and wake model used in this 
work, for the wind scenario 1 (0 deg wind direction), the transport delays $\tau_{i, j}(t)$ can be estimated as follows:

$$
\begin{aligned}
& f\left(D_{i, 1}, v_{w}^{0}(t)\right)=\frac{10 L}{v_{w}^{0}(t)}, \quad \forall i=1,2,3 \\
& f\left(D_{i, 2}, v_{w}^{0}(t)\right)=\frac{10 L}{v_{w}^{0}(t)}+\frac{7 L}{0,9 v_{w}^{0}(t)}, \quad \forall i=1,2,3 \\
& f\left(D_{i, 3}, v_{w}^{0}(t)\right)=\frac{10 L}{v_{w}^{0}(t)}+\frac{7 L}{0,9 v_{w}^{0}(t)}+\frac{7 L}{0,81 v_{w}^{0}(t)}, \quad \forall i=1,2,3
\end{aligned}
$$

Redundancy equations (9)-(11) can be discretized and expressed in regression form as (6):

$$
\begin{array}{r}
\hat{P}_{i, j}(k)=F_{P_{i, j}}\left(k, d_{i, j}\left(\mathbf{p}_{k}\right), \boldsymbol{\theta}_{P_{i, j}}\left(\mathbf{p}_{k}\right), P_{r_{i, j}}(k)\right) \\
\hat{\beta}_{i, j}(k)=F_{\beta_{i, j}}\left(k, d_{i, j}\left(\mathbf{p}_{k}\right), \boldsymbol{\theta}_{\beta_{i, j}}\left(\mathbf{p}_{k}\right), P_{r_{i, j}}(k)\right) \\
\hat{\omega}_{g_{i, j}}(k)=F_{\omega_{g i, j}}\left(k, d_{i, j}\left(\mathbf{p}_{k}\right), \boldsymbol{\theta}_{\omega_{g i, j}}\left(\mathbf{p}_{k}\right), P_{r_{i, j}}(k)\right)
\end{array}
$$

with $F_{P_{i, j}}, F_{\beta_{i, j}}$ and $F_{\omega_{g i, j}}$ nonlinear discrete functions of outputs $\hat{P}_{i, j}(k), \hat{\beta}_{i, j}(k)$ and $\hat{\omega}_{g i, j}(k)$ respectively, with the same input $P_{r_{i, j}}(k)$. Parameters $\boldsymbol{\theta}_{P_{i, j}}\left(\mathbf{p}_{k}\right), \boldsymbol{\theta}_{\beta_{i, j}}\left(\mathbf{p}_{k}\right)$ and $\boldsymbol{\theta}_{\omega_{g i, j}}\left(\mathbf{p}_{k}\right)$ vary with the same scheduling variables:

$\mathbf{p}_{k}=\left(v_{w}^{0}(k), v_{w}(k)\right)^{T}$

Finally, $d_{i, j}\left(\mathbf{p}_{k}\right)$ is the discrete time delay that can be computed as:

$$
d_{i, j}\left(\mathbf{p}_{k}\right)=\left[\frac{f\left(D_{i, j}, v_{w}^{0}(k)\right)}{T_{s}}\right]
$$

Remark: the filtered wind measurement $v_{w}^{0}(k)$ is used for delay estimation (13) and the direct wind measurement $v_{w}(k)$ (without filtering) is used for the wind estimation (12).

Subtracting estimations (15)-(17) from real data measurements, three residuals are obtained for every wind turbine, resulting in 27 residuals. The main drawback of these 27 temporal parity equations is that they depend directly on the scheduling variable which, in this case, is the wind that is measured with a high noise level, leading to a big wind speed estimation error bound $\sigma_{w_{i, j}}$ in (12). On the other hand, the spatial redundancy is based on comparing those wind turbines that receive the same wind. This allows generating other relations that are less dependent on the wind measurements. For example, in wind scenario 1 (wind direction $0 \mathrm{deg}$ ) the wind for all the wind turbines that are in the same $y$-column (see Fig. 1) is expected to be the same at every instant $k$ that implies:

$$
v_{w_{i}}(k)=v_{w_{1, i}}(k)=v_{w_{2, i}}(k)=v_{w_{3, i}}(k) \quad i=1, \ldots, 3 \quad \forall k
$$

Then, if the reference power is the same for all the generators in the wind farm, as it usually happens or can be imposed for a while for fault detection purposes, the following topological relations can be deduced:

$$
\begin{array}{ll}
P_{1, i}(k)=P_{2, i}(k)=P_{3, i}(k) & i=1, \ldots, 3 \quad \forall k \\
\beta_{1, i}(k)=\beta_{2, i}(k)=\beta_{3, i}(k) & i=1, \ldots, 3 \quad \forall k \\
\omega_{g_{1, i}}(k)=\omega_{g_{2, i}}(k)=\omega_{g_{3, i}}(k) & i=1, \ldots, 3 \quad \forall k
\end{array}
$$

In a real scenario (19) would include some noise:

$$
\begin{aligned}
& v_{w_{1, i}}(k)=v_{w_{i}}(k)+e_{w_{1, i}}(k), \quad\left|e_{w_{1, i}}(k)\right| \leq \sigma_{w_{i}} \\
& v_{w_{2, i}}(k)=v_{w_{i}}(k)+e_{w_{2, i}}(k), \quad\left|e_{w_{2, i}}(k)\right| \leq \sigma_{w_{i}} \\
& v_{w_{3, i}}(k)=v_{w_{i}}(k)+e_{w_{3, i}}(k), \quad\left|e_{w_{3, i}}(k)\right| \leq \sigma_{w_{i}}
\end{aligned}
$$

and (20) would lead to:

$$
\begin{aligned}
& P_{1, i}(k)-P_{2, i}(k)=\hat{P}_{1, i}(k)-\hat{P}_{2, i}(k)+e_{P_{1-2, i}}(k) \\
& P_{1, i}(k)-P_{3, i}(k)=\hat{P}_{1, i}(k)-\hat{P}_{3, i}(k)+e_{P_{1-3, i}}(k) \\
& P_{2, i}(k)-P_{3, i}(k)=\hat{P}_{2, i}(k)-\hat{P}_{3, i}(k)+e_{P_{2-3, i}}(k)
\end{aligned}
$$

In this way, 9 spatial relations between the power wind turbine magnitudes can be obtained in wind scenario 1 . The same relations are obtained for the pitch and the angular velocity. This means that 27 additional parity equations are added to the 27 temporal parity equations. On the other hand, in wind scenario 2 the following wind speed ideal relations can be deduced:

$$
\begin{gathered}
v_{w_{2,1}}(k)=v_{w_{1,2}}(k), \quad v_{w_{3,2}}(k)=v_{w_{2,3}}(k) \quad \forall k \\
v_{w_{3,1}}(k)=v_{w_{2,2}}(k)=v_{w_{1,3}}(k)
\end{gathered}
$$

Hence, 15 additional spatial parity equations can be obtained.

The main advantage of spatial versus temporal relations is that spatial relations are not affected by uncertainty in transport delay $\lambda_{\tau_{i j}}$ (13).

Remark: As spatial relations depend on the wind direction, they have to be reconfigured according to this wind direction provided by a sensor or estimated with the available measurements.

\subsection{Fault isolation}

Fault 1 influences the power residuals, Fault 2 influences the pitch angle residuals and, finally, Fault 3 influences the power and the generator speed residuals.

The effects of Fault 3 are the increase of the amplitude of a $10 \mathrm{~Hz}$ sine function, while Faults 1 and 2 are low frequency faults. This fact, and the need of filtering the residuals in order to decrease the effect of measurement noises, lead to filter power residuals with low pass $(L P)$ and band pass $(B P)$ filters so as to better detect Fault 1 and Fault 3, respectively. On the other hand, low pass and band pass filters have been used in pitch angle and speed residuals, respectively, in order 
to enhance the detectability of Faults 2 and 3 respectively.

Table 1 shows the theoretical signature matrix that can be derived from the temporal residuals of a particular wind turbine. As can be deduced from Table 1, temporal residuals allow detecting and isolating the proposed 3 faults for each wind turbine. Moreover, the extra information provided by the spatial residuals increases the robustness in the detectability and isolability, as illustrated in Table 2, where the power residuals (LP filtered) of column 1 (wind scenario 1) extracted from (24) are related to the Fault 1 of the wind turbines of this column.

Table 1. Signature matrix temporal residuals

\begin{tabular}{|c|c|c|c|}
\hline Residuals & Fault 1 & Fault 2 & Fault 3 \\
\hline$P_{L P}$ & $\mathrm{X}$ & & \\
\hline$P_{B P}$ & & & $\mathrm{x}$ \\
\hline$\beta_{L P}$ & & $\mathrm{x}$ & \\
\hline$\omega_{g B P}$ & & & $\mathrm{X}$ \\
\hline
\end{tabular}

Table 2. Signature matrix spatial residuals $P_{L P}$ column 1 wind case 1 ( 0 deg)

\begin{tabular}{|c|c|c|c|}
\hline Residuals & $\begin{array}{c}\text { Fault 1 } \\
\text { turbine 1,1 }\end{array}$ & $\begin{array}{c}\text { Fault 1 } \\
\text { turbine 2,1 }\end{array}$ & $\begin{array}{c}\text { Fault 1 } \\
\text { turbine 3,1 }\end{array}$ \\
\hline$P_{L P_{1,1}}(k)-P_{L P_{2,1}}(k)$ & $\mathrm{x}$ & $\mathrm{x}$ & \\
\hline$P_{L P_{1,1}}(k)-P_{L P_{3,1}}(k)$ & $\mathrm{X}$ & & $\mathrm{X}$ \\
\hline$P_{L P_{2,1}}(k)-P_{L P_{3,1}}(k)$ & & $\mathrm{X}$ & $\mathrm{X}$ \\
\hline
\end{tabular}

\section{RESULTS}

In the following, the results obtained applying the methodologies described above to the realistic benchmark implemented in Matlab-Simulink ${ }^{\odot}$ and available in: http://www.kk-electronic.com/Default.aspx?ID=9593 are presented.

\subsection{Model Calibration}

Because of space limitation, only the results obtained in wind scenario 1 (0 deg) are illustrated. Applying $4400 \mathrm{~s}$ long wind sequences to the Matlab-Simulink ${ }^{\complement}$ benchmark, a set of input/output data $P_{r_{i, j}}(t), P_{i, j}(k), \quad \beta_{i, j}(k)$ and $\omega_{g i, j}(k)$, $k=1, \ldots, N$ has been obtained.

At first, the three different wind speed estimation sequences $\left(\hat{v}_{w_{1}}, \hat{v}_{w_{2}}\right.$ and $\left.\hat{v}_{w_{3}}\right)$ have been computed applying (12) to the wind speed provided by the measuring mast and considering transport delays (14). Fig. 2 shows the real sequence of wind speed in turbine 1,1 $\left(v_{w_{1,1}}\right)$ and the one estimated for all the turbines in the $y$-column 1 i.e. $\hat{v}_{w_{1}}=\hat{v}_{w_{11}}=\hat{v}_{w_{21}}=\hat{v}_{w_{31}}$ (see Fig. 1). Delay uncertainties $\lambda_{\tau_{1}}$, $\lambda_{\tau_{2}}$ and $\lambda_{\tau_{3}}$ have been computed following the signal processing method described in Blesa et al. (2010). Uncertainty bounds $\sigma$ of wind speed error estimation (12) and noise (23) have been obtained by a noise analysis of the error between the wind speed variables and estimations.

Continuous functions (9)-(11) have been obtained by means of the physical knowledge of the system and discretized with $T_{s}=0.01 \mathrm{~s}$. Then, the nominal parameters $\boldsymbol{\theta}_{P_{i, j}}^{0}\left(\mathbf{p}_{k}\right), \boldsymbol{\theta}_{\beta_{i, j}}^{0}\left(\mathbf{p}_{k}\right)$ and $\boldsymbol{\theta}_{\omega_{g i, j}}^{0}\left(\mathbf{p}_{k}\right)$ have been obtained and 54 parity equations (27 temporal and 27 spatial) have been defined. $L P$ and $B P$ filters have been designed in order to minimize the effect of the noise that has been bounded in each parity equation (uncertainty bounds $\sigma$ in temporal and spatial parity equations). Finally, an optimization problem as proposed in Blesa et. al. (2014) has been applied, in order to compute a tolerance $\alpha$ in parameters of each parity equation $\left(\lambda_{i}(k)=\alpha \theta_{i}^{0}\left(\mathbf{p}_{k}\right), \quad i=1, \ldots, n_{\theta}\right) \quad$ such as the predicted behavior contains all the data in the fault free scenario (5).

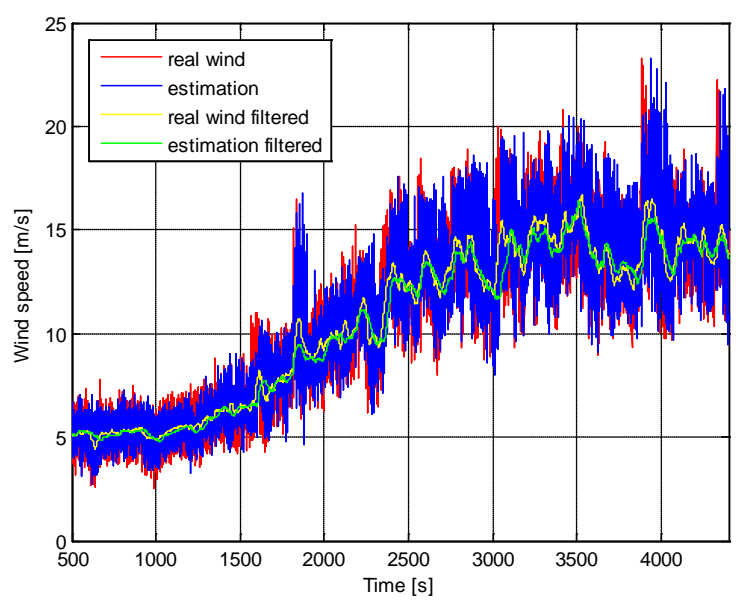

Fig. 2. Real and estimated wind speed in turbine 1,1 .

Fig. (3) shows the evolution of the power spatial residual $P_{1,2}-P_{2,2}$ with its interval bounds in the fault free scenario used for the model calibration.

As it can be observed from Fig. 3, the residual interval bounds vary strongly with the operating point in the power spatial residual. This is due to the fact that small differences in the wind speed produce different effects in the power generation. These differences are much lower in the pitch residual and negligible in the angular speed residual (the corresponding figures are omitted due to lack of space).

The same procedure has been carried out to calibrate the 42 parity equations (27 temporal and 15 spatial) for wind scenario 2 (45 deg). In this scenario, 5 different wind speed estimation sequences, corresponding to the number of wind turbine sets that are supposed to receive the same wind speed, have been estimated.

\subsection{Fault scenarios}

The proposed FDI approach is tested in Fault 1 (the debris build-up on the blades), Fault 2 (pitch misalignment) and Fault 3 (decrease in drive train damping) cases that are described in Section 3.2. These faults are occurring twice in three different wind turbines at different time intervals, i.e. no multiple faults are present at any time. All faults occur once before $2300 \mathrm{~s}$ (a) and once after $2300 \mathrm{~s}$ (b). In the first period, the wind farm cannot deliver the required power, while it can do it in the second one. Fig. 4 shows, for fault scenario 1(b), the evolution of spatial residual $P_{1,2}-P_{3,2}$ that is sensitive to this fault in wind scenario 1. 


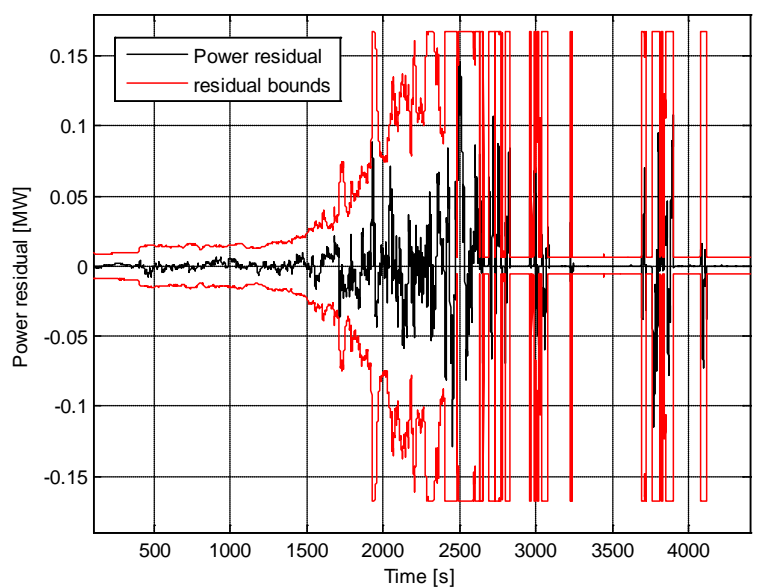

Fig. 3. $P_{1,2}-P_{2,2}$ residual and bounds in fault free scenario.

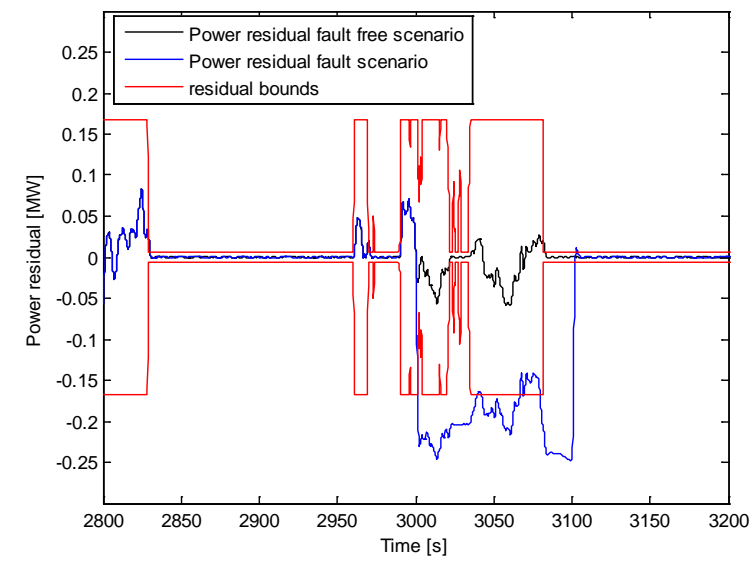

Fig. 4. Evolution of residual $P_{1,2}-P_{3,2}$ and bounds in fault scenario 1(b): Debris build-up the wind turbine blades lowering maximum power $6 \%$ in wind turbine 1,2 from $\mathrm{t}=3000$ s to $\mathrm{t}=3100$ s.

Fault detection and isolation results in wind scenarios 1 and 2 of the proposed FDI method implemented in MatlabSimulink ${ }^{\complement}$, have been obtained after an exhaustive Monte Carlo analysis where the condition of false positive detections proposed in Odgaard and Stoustrup (2013) has been verified. In general, the behavior of the FDI method is better in the wind scenario 1 than in wind scenario 2 due to the higher number of spatial relations (27 in wind scenario 1 and 15 in wind scenario 2). Fault 1 (Debris build-up) is detected better in case (b) (after 2300s) due to the fact that the available power is higher and the scaling effect is more important than in case (a) (before 2300s), where the available power is lower. Fault 2 (pitch misalignment) is better detected in case (a) because the uncertainty is slightly lower than in case (b). In order to have a sensitivity of $0.3^{\circ}$ in fault detection in this fault, a very restrictive low-pass filter has been implemented due to the pitch sensor noise. Then, despite the FDI block can detect and isolate $0.3^{\circ}$ in misalignment, the magnitude has to be increased to $10^{\circ}-14^{\circ}$ in order to guarantee the correct detection and isolation in less than 3s as was proposed in Odgaard and Stoustrup (2013). Finally, Fault 3 (decrease in drive train damping) is detected and isolated correctly (Odgaard and Stoustrup, 2013).

\section{CONCLUSIONS}

In this paper, the problem of the fault diagnosis of a wind farm has been addressed using interval parity equations and NLPV models. Fault detection is based on the use of parity equations and unknown but bounded description of the noise and modeling errors. The fault detection test is based on checking the consistency between the measurements and the model by finding if the measurements are inside the interval prediction bounds. The fault isolation algorithm is based on analyzing the observed fault signatures on-line, and matching them with the theoretical ones obtained using structural analysis. Finally, the proposed approach has been tested using the wind farm benchmark proposed in the context of the wind farm FDI/FTC competition obtaining satisfactory results.

\section{REFERENCES}

Blanke M., Kinnaert M., Lunze J. and Staroswiecki M. (2006). Diagnosis and Fault-Tolerant Control. Springer, 2nd edition.

Blesa J., Puig V. and Bolea Y. (2010). "Fault detection using interval LPV models in an open-flow canal", Control Engineering Practice, 18(5), pp. 460-470.

Blesa, J., Rotondo, D., Puig, V., Nejjari, F. (2014). "FDI and FTC of windcturbines using the interval observer approach and virtual actuators/sensors". Control Engineering Practice 24(2014) 138-155.

Chen J. and Patton R. J. (1999). Robust Model-Based Fault Diagnosis for Dynamic Systems, Kluwer Academic Publishers, Boston, MA.

Fagarasan, I., Ploix S. and Gentil S. (2004). "Causal fault detection and isolation based on a set-membership approach", Automatica, 40(12), pp. 2099-2110.

Isermann, R. (2005). "Model-based fault-detection and diagnosis status and applications”, in Annual Reviews in Control, 29(1), pp. 71-85.

Johnson, K. E. Pao L. Y., Balas M. J. and Fingersh L. J. (2006). "Control of variable-speed wind turbines: standard and adaptive techniques for maximing energy capture”, IEEE Control Systems Magazine, 26(3), pp. 70-81.

Odgaard P. F., Bækgaard M. and Astrup B. (2010). "Model based control of wind parks", in Proceedings of EWEC 2010. Warsaw, Poland, April 2010.

Odgaard P. F., Stoustrup J. and Kinnaert M. (2013). "Fault tolerant control of wind turbines - a benchmark model”, IEEE Transactions on Control Systems Technology, 21(4), pp. 11681182.

Odgaard. P. F. and Stoustrup J. (2013). "Fault tolerant wind farm control - a benchmark model”. In Proceedings of the 2013 IEEE Multi-conference on Systems and Control, Hyderabad, India, August 2013.

Patton R. J. and Chen J. (1994). "A review of parity space approaches to fault diagnosis for aerospace systems”, Journal of Guidance, Control and Dynamics, 17(2), pp. 278-285.

Previdi F. and Lovera M. (2004). "Identification of non-linear parametrically varying models using separable least squares", International Journal of Control, 77(16), pp. 1382-1392.

Puig V., Quevedo J., Escobet T., Nejjari F. and De las Heras S. (2008). "Passive Robust Fault Detection of Dynamic Processes Using Interval Models”, IEEE Transactions on Control Systems Technology, 16(5), pp. $1083-1089$.

Sainz, M. Á. Armengol J. and Vehí J. (2002). "Fault detection and isolation of the three-tank system using the modal interval analysis”, Journal of Process Control, 12(2), pp. 325-338. 